\title{
Etude Phytochimique Et Activité Antibactérienne De Deux Espèces De Lavande Autochtones Au Maroc : «Lavandula stoechas L. et Lavandula dentata L.»
}

\author{
Lamiae Bachiri \\ Equipe de Microbiologie du Sol et de l'Environnement, \\ Université Moulay Ismail, Faculté des Sciences, Meknès, Maroc \\ Ghizlane Echchegadda \\ Département de la Protection de Plantes et de l'Environnement, \\ Ecole Nationale d'Agriculture, Meknès, Maroc \\ Jamal Ibijbijen \\ Laila Nassiri \\ Equipe de Microbiologie du Sol et de l'Environnement, \\ Université Moulay Ismail, Faculté des Sciences, Meknès, Maroc \\ doi: 10.19044/esj.2016.v12n30p313 URL:http://dx.doi.org/10.19044/esj.2016.v12n30p313
}

\begin{abstract}
In the present work, the aim is to compare the chemical composition and antibacterial activity of two lavender Species "Lavandula stoechas L." and "Lavandula dentata L.", belonging to wild flora of Morocco.

Thus, a phytochemical screening was performed on the aerial part of invested plants, and no qualitative difference was detected between the studied species. Indeed, both contain catechin and gallic tannins, flavonoids (anthocyanins, flavones and catechols), sterols and triterpenes, in addition to combined anthracene compounds (C-glycosides). Whereas, the dosage of crude aqueous extracts showed that the contents of secondary metabolites, such as polyphenols, flavonoids and total and condensed tannins were higher in L. dentata.

Also, the essential oils of the aerial part, obtained by hydrodistillation gave higher yields in L. dentata than $L$. stoechas.

Finally, antibacterial activity tests were performed on four strains of bacterial pathogens (Staphylococcus aureus "Gram+," and Escherichia coli; Klebsiella pneumoniae and Proteus mirabilis which are Gram -) with both essential oils and crude aqueous extracts. The results obtained showed that essential oils have a powerful effect, particularly that of $L$. dentata on $S$. aureus, with an inhibition diameter about $30 \mathrm{~mm}$. The crude extracts showed moderate antibacterial activity against Gram - and had no effect on Gram +.
\end{abstract}


Keywords: Lavandula dentata, Lavandula stoechas, phytochemistry, extracts, essential oil, antibacterial activity

\section{Résumé}

Dans le présent travail, il s’agit de comparer la composition chimique et le pouvoir antibactérien de deux espèces de lavande "Lavandula stoechas L." et "Lavandula dentata L.", appartenant à la flore spontanée du Maroc.

Ainsi, un criblage phytochimique a été réalisé sur la partie aérienne des plantes investies; aucune différence qualitative ne fut décelée entre les espèces étudiées. En effet, toutes les deux renferment des tanins catéchiques et galliques, des flavonoïdes (anthocyanes, flavones et catéchols), des stérols et triterpènes, en plus de composés anthracéniques combinés ( $\mathrm{C}$ hétérosides). Par contre, le dosage des extraits aqueux bruts a montré que les teneurs en métabolites secondaires, notamment les polyphénols, les flavonoïdes et les tanins totaux et condensés étaient plus élevées chez $L$. dentata.

De leur côté, les huiles essentielles de la partie aérienne, obtenues par hydrodistillation ont donné des rendements plus importants chez L dentata par rapport à $L$. stoechas.

En fin, des tests d'activité antibactérienne ont été réalisés sur quatre souches bactériennes pathogènes (Staphylococcus aureus «Gram+ » et Escherichia coli, Klebsiella pneumoniae, et Proteus mirabilis qui sont des Gram-), à la fois avec les huiles essentielles et les extraits aqueux bruts. Les résultats obtenus ont montré que les huiles essentielles ont un effet puissant, notamment celle de $L$. dentata sur $S$. aureus, avec un diamètre d'inhibition allant jusqu’à $30 \mathrm{~mm}$. Quant aux extraits bruts, ils ont montré une activité antibactérienne modérée contre les bactéries Gram (-) et n’ont eu aucun effet sur les Gram (+).

Mots clés : Lavandula dentata- Lavandula stoechas- Phytochimie- ExtraitsHuile essentielle- Activité antibactérienne.

\section{INTRODUCTION}

Le genre Lavandula, affilié à la sous-famille des Népétoïdées (Dupont et Guignard, 2012) est composé d'environ 39 espèces, de nombreux hybrides, et près de 400 cultivars enregistrés (Upson et Anderws, 2004). La plupart des espèces, de souche méditerranéenne, sont très aromatiques et sont employées comme expectorant, antispasmodique, désinfectant des plaies, antimicrobien, anti-carcinogène, sédatif, antidépresseur, antioxydant, antiinflammatoire et insecticide (Chu et al., 2007 ; Gören et al., 2002).

Au Maroc, en plus d'espèces cultivées, notamment le lavandin hybride, plusieurs autres y abondent à l'état sauvage dans différentes conditions 
pédologiques et sous divers bioclimats méditerranéens, des plus sévères comme le saharien jusqu'au subhumide, voire l'humide (Bellakhdar, 1997). D'un autre côté, chez plusieurs espèces aromatiques et médicinales, quoique groupées sous le même nom vernaculaire ou commun, des différences sont enregistrées aussi bien au niveau intra qu'interspécifique quant à leur composition chimique quantitative et qualitative. Cela peut se répercuter sur leur activité biologique qui, sans aucun doute, est corrélée à cette composition. Par exemple, au sein du thym, l'espèce Thymus ciliatus (Desf.) Benth voit son huile essentielle riche en thymol (44,2 \%), $\beta$-E-ocimène $(25,8$ $\%$ ) et $\alpha$-terpinène $(12,3 \%)$ alors que Thymus algeriensis Boiss. \& Reut est plutôt riche en camphre $(27,7 \%)$ et en $\alpha$-pinène $(20,5 \%)$; testées sur Bacillus subtilis, Escherichia coli, Micrococcus luteus et Staphylococcus aureus, les huiles essentielles du T.ciliatus se sont révélées plus bioactives, probablement en rapport avec leur richesse en thymol, connu pour son efficacité contre les agents microbiens (Amarti et al., 2010). C'est également dans cette optique que s'encarte le présent travail ; en effet, notre objectif majeur est la comparaison de deux espèces de lavande, autochtones au Maroc : Lavandula dentata et Lavandula stoechas.

\section{MATERIEL ET METHODES}

\section{Récolte et Identification du matériel végétal}

Plusieurs sorties ont été organisées durant l’année 2015 dans différentes régions du Maroc, en quête de lavandes spontanées; ainsi, une première espèce fut repérée et récoltée au plateau central « Oulmès »; une autre espèce a été récoltée au niveau du haut Atlas occidental « Marrakech ». Les échantillons récoltés ont été identifiés par l’équipe de botanique de la Faculté des sciences de Meknès en se référant au manuel de systématique de floristique (Fennane et al., 2007).Elles correspondent respectivement à Lavandula dentata et Lavandula stoechas.

À chaque fois les coordonnées des sites d'échantillonnage ont été calculés et définies par le système GPS (Tableau 1)

Tableau 1 : Bioclimats et Substrats des sites de récolte

\begin{tabular}{|c|c|c|c|c|c|c|c|}
\hline Espèces & Localité & $\begin{array}{c}\text { Site de } \\
\text { collection }\end{array}$ & $\begin{array}{c}\text { Zone } \\
\text { bioclimatiq } \\
\text { ue }\end{array}$ & Substrat & & \multicolumn{2}{|c|}{ Coordonnées géographiques } \\
\cline { 5 - 7 } & & Oulmès & $\begin{array}{c}\text { Subhumide } \\
\text { à humide } \\
\text { tempéré- } \\
\text { frais }\end{array}$ & Silice & $33^{\circ} 25^{\prime} 0.01^{\prime \prime}$ & $6^{\circ} 1^{\prime} 0.01^{\prime \prime}$ & 1250 \\
$\begin{array}{c}\text { Lavandula } \\
\text { spp. } \\
\text { Stoechas }\end{array}$ & $\begin{array}{c}\text { Plateau } \\
\text { d'Oulmès }\end{array}$ & On) & $\begin{array}{c}\text { Longitude } \\
\text { (E) }\end{array}$ & Altitude (m) \\
\hline $\begin{array}{c}\text { Lavandula } \\
\text { dentata } \\
\text { spp.dentata }\end{array}$ & $\begin{array}{c}\text { Haut Atlas } \\
\text { occidental }\end{array}$ & $\begin{array}{c}\text { Bassin } \\
\text { d'Amassine }\end{array}$ & $\begin{array}{c}\text { semi-aride } \\
\text { supérieur } \\
\text { tempéré }\end{array}$ & $\begin{array}{c}\text { Argile } \\
\text { rouge et } \\
\text { pélites }\end{array}$ & $31^{\circ} 18^{\prime} 36^{\prime \prime}$ & $7^{\circ} 46^{\prime} 48^{\prime \prime}$ & 553 \\
\hline
\end{tabular}




\section{Détermination du taux d'humidité}

La teneur en eau des plantes a été déterminée par le procédé de séchage à l'étuve, à $105^{\circ} \mathrm{C}$ (Twidwell et al., 2002). Pour chaque espèce, $100 \mathrm{~g}$ de la matière fraiche ont ainsi été étuvés pendant une heure ; trois répétitions ont été réalisées pour chaque échantillon, dont la moyenne représenterait le taux d'humidité.

Préparation des extraits aqueux et extraction des huiles essentielles

L’infusé, le décocté et le macéré ont été préparés selon la méthode adoptée par Sqalli et al. (2007), utilisant $10 \mathrm{~g}$ de poudre pour $100 \mathrm{ml}$ d'eau. Le rendement des différents extraits est calculé par la formule : Rendement ext $\%=\mathbf{M}$ ext $/ \mathbf{M}$ éch $\times 100$, avec :

$\mathbf{M}$ ext : Masse de l'extrait après évaporation du solvant en $\mathrm{g}$

M éch : Masse de l'échantillon végétal en g

De son côté, l'extraction des huiles essentielles a été effectuée par hydrodistillation avec un appareil de type Clevenger (Clevenger, 1928). Ainsi, trois distillations ont été réalisées dans un ballon, par ébullition durant quatre heures, de $100 \mathrm{~g}$ de matériel végétal frais avec un litre d'eau.

\section{Rendement $\mathbf{H E}=\mathbf{M}^{\prime} / \mathbf{M} \times \mathbf{1 0 0}$, avec :}

M': Masse de l'huile essentielle obtenue en $\mathrm{g}$;

M : Masse de la matière végétale sèche utilisée en g.

\section{Screening phytochimique}

L’étude phytochimique qualitative permet de détecter les différentes familles chimiques constituants une plante; les tests y sont basés sur l'intensité du précipité et de la turbidité, la coloration étant proportionnelle à la quantité de substances recherchées.

Aussi, avons-nous procédé à la caractérisation des métabolites secondaires présentant un intérêt pharmacologique tels les stérols et les triterpènes, les tanins, les flavonoïdes, les alcaloïdes, les saponosides et les composés réducteurs; ceci, en se référant aux techniques décrites par Bruneton, 1993 ; Dohou, 2003 ; Bekro et al, 2007.

Ainsi, la présence des alcaloïdes est mise en évidence grâce aux réactifs de Mayer et de Dragendorff ; celle des tanins, par une solution aqueuse de trichlorure ferrique $\left(\mathrm{Fecl}_{3}\right)$ et des tanins galliques et catéchiques, par le réactif de Stiasny.

Les composés appartenant au groupe des flavonoïdes sont révélés par la réaction à la cyanidine. La présence des dérivés anthracéniques est révélée à l'aide d'un résidu de poudre de la plante épuisée par chloroforme $\left(\mathrm{CHCl}_{3}\right)$, puis, une solution d'hydroxyde d'ammonium $\left(\mathrm{NH}_{4} \mathrm{OH}\right)$ diluée à $50 \%$ qui, elle, permet de mettre en évidence les deux formes hétérosidiques (Ohétérosides et C-hétérosides). 
Quant à la détection des Stérols et des triterpènes, elle est réalisée par la réaction de Liebermann Buchard, et celle des saponosides par leur pouvoir moussant en solution aqueuse.

En fin, la révélation des oses et holosides est réalisée par l'éthanol saturé avec du thymol et celle des mucilages, par l’éthanol absolu uniquement.

\section{Analyse quantitative des extraits Dosage des polyphénols totaux :}

Les polyphénols ont été déterminés par la méthode du FolinCiocalteu, décrite par Vermerius et al., (2006) qui permet de connaitre le contenu polyphénolique total d'un échantillon donné. Son principe consiste à mélanger $0.1 \mathrm{ml}$ de l'extrait, avec $2 \mathrm{ml}$ d'une solution de carbonate de sodium à $2 \%$; après agitation et incubation pendant 5 minutes, $0.1 \mathrm{ml} \mathrm{du}$ réactif Folin- Ciocalteu $(1 \mathrm{~N})$ est ajouté. Le mélange obtenu est incubé à température ambiante, à l'abri de la lumière pendant 30 minutes ; l'absorbance est ensuite mesurée à l'aide d'un spectrophotomètre à $700 \mathrm{~nm}$.

Les tests ont été effectués trois fois pour une fiabilité des résultats. La quantification des polyphénols totaux a été faite à l'aide d'une courbe d'étalonnage, réalisée par l'acide gallique à différentes concentrations et dans les mêmes conditions que les échantillons.

Les résultats sont exprimés en mg équivalent d’acide gallique par g d'extrait.

\section{Dosage des flavonoïdes :}

La détermination du taux de flavonoïdes totaux de l'extrait a été réalisée par le protocole modifié de Kumaran et Karunakaran, 2005. $1 \mathrm{ml}$ de l'extrait de plante est mélangé avec $1 \mathrm{ml}$ d'une solution du trichloride d'aluminium (2\%) et une goutte d'acide acétique. L'absorbance a été mesurée à $415 \mathrm{~nm}$, après incubation à température ambiante pendant $40 \mathrm{~min}$. Dans les mêmes conditions, la solution standard de quercétine a été préparée avec une concentration de $0,1 \mathrm{mg} / \mathrm{ml}$. Toutes les manipulations ont été répétées 3 fois.

\section{Dosage des tanins totaux :}

Les tanins totaux ont été estimés en mélangeant $100 \mathrm{mg}$ de polyvinylpolypyrrolidone (PVPP) avec $2 \mathrm{ml}$ d'eau distillée et $2 \mathrm{ml}$ d'extrait. L'ensemble fut agité et maintenu à $4^{\circ} \mathrm{C}$ pendant $15 \mathrm{~min}$, puis centrifugé à $3000 \mathrm{~g}$, à $4^{\circ} \mathrm{C}$ pendant $10 \mathrm{~min}$. Le surnageant, qui en a résulté, fut utilisé pour la détermination des tanins totaux qui correspondent à la différence entre les phénols totaux (mesurés par le procédé de Folin-Ciocalteau) avant et après traitement avec le PVPP (http://www.iaea.org/programmes/nafa/d3/index.html) 


\section{Dosage des tanins condensés :}

Les tanins condensés ont été déterminés en milieu acide, par la méthode de la vanilline, décrite par Ba et $\boldsymbol{a l . , ~ 2 0 1 0}$. Le réactif de vanilline fut préparé en mélangeant à volume égal : l'acide chloridrique à $8 \%(\mathrm{v} / \mathrm{v})$, le méthanol à $37 \%(\mathrm{v} / \mathrm{v})$ et $4 \%$ de vanilline dans du méthanol $(\mathrm{m} / \mathrm{v})$. Le mélange fut maintenu à $30^{\circ} \mathrm{C}$ avant le dosage.

$200 \mu \mathrm{l}$ de chaque extrait à analyser ont été ajoutés à $1000 \mu \mathrm{l}$ du réactif de vanilline ; le mélange, agité, a été incubé pendant 20 min à $30{ }^{\circ} \mathrm{C}$ et à l'obscurité. L'absorbance fut ensuite mesurée à $500 \mathrm{~nm}$ par un spectrophotomètre UV, contre un blanc constitué d'un mélange de méthanol (37\%) et d’acide chloridrique (8\%) à volume égal.

\section{Activité antibactérienne des huiles essentielles et des extraits aromatiques}

Tests de l'activité antibactérienne (Méthode de diffusion ou de l'aromatogramme)

Les souches bactériennes choisies pour cette étude sont des bactéries pathogènes; il s'agit de trois bactéries Gram négatives (Escherichia coli, Klebsiella pneumoniae et Proteus mirabilis) et une bactérie Gram positive (Staphylococcus aureus). Ces souches, toutes issues de prélèvements hospitaliers à l'hôpital Mohamed V Meknès, sont réputées être à l'origine de plusieurs infections (urinaire, intestinale, respiratoire, etc...)...

Pour étudier l'activité antibactérienne des huiles essentielles et des extraits aqueux bruts obtenus, on a choisi la méthode de diffusion sur disques, appelée aussi méthode de Vincent ou technique de l'aromatogramme mise au point par Schroeder et Messing en 1949 et cité par (Rhayour, 2002). Dans cette méthode, on a utilisé des disques de papier Wattman de $6 \mathrm{~mm}$ de diamètre, imprégnés par un volume connu de l'huile essentielle ou de l'extrait brut à évaluer et déposés à la surface d'un milieu gélosé (Agar de Muller Hinton «AMH»), préalablement ensemencé par écouvillonnage en surface par la suspension bactérienne. Après incubation pendant $24 \mathrm{~h}$ à $37^{\circ} \mathrm{C}$, la lecture des résultats a été faite par la mesure du diamètre de la zone d'inhibition en mm (Ponce et al., 2003).

Détermination des concentrations minimale inhibitrice (CMI) et bactéricide (CMB) pour les huiles essentielles et les extraits aqueux bruts

-La Concentration Minimale Inhibitrice (CMI) est définie comme étant la plus petite dilution dans laquelle aucune croissance macroscopique n'est observée (Kuete et al., 2004).

-La Concentration Minimale Bactéricide (CMB) correspond à la plus faible concentration en huile essentielle ou en extrait brut 
capable de tuer plus de 99,9 \% de l’inoculum bactérien initial (soit moins de $0,01 \%$ de survivants).

Ainsi, dans une série de tubes à hémolyse, un volume constant $(4 \mathrm{ml})$ du bouillon nutritif (BMH) déjà stérilisé, fut réparti avec un inoculum fixe de bactérie $(50 \mu \mathrm{l})$, d'une culture pure de 18 à 24 heures, dont la densité bactérienne est équivalente au standard Mc Farland 0,5 ( 108 UFC/ml).

Puis, une solution mère $(50 / 50 \mu \mathrm{l})(\mathrm{V} / \mathrm{V})$ fut préparée sur la base de volumes égaux de l'huile essentielle et du Tween 80. Par la suite, des concentrations croissants d'huile $(4.95 ; 6.93 ; 8.91 ; 11.88$ et $13.36 \mathrm{mg} / \mathrm{ml})$ et d'extraits aqueux $(60 ; 70 ; 80 ; 90 ; 100 ; 110 \mathrm{mg} / \mathrm{ml})$ ont été ajoutés aux tubes d'hémolyse suscités. Un tube témoin fut laissé sans extrait et sans huile essentielle. Après incubation à $37^{\circ} \mathrm{C}$ pendant 24 heures, les tubes ont été soigneusement examinés. En principe, les tubes témoins sont uniformément troubles et le premier tube de la série demeuré limpide indique précisément la CMI.

Pour la CMB, elle est déterminée par ensemencement des prélèvements qui sont effectués dans le tube témoin et dans chacun des tubes limpides puis déposés « en strie » sur gélose MHA ; les boîtes ainsi ensemencées, sont incubées durant 24 heures à $37^{\circ} \mathrm{C}$ et la $\mathrm{CMB}$ correspond à la plus petite concentration qui ne donne aucune subculture.

Analyse statistique : Les données brutes recueillies ont été reportées sur une base de données puis traitées et analysées statistiquement à l’aide du logiciel « Excel, 2013 ».

\section{RESULTATS ET DISCUSSION Taux d'humidité}

Le taux d'humidité correspond à environ $62.6 \%$ pour L. dentata et $64 \%$ pour L. stoechas, et donc plus de la moitié du poids frais des deux espèces est constituée d'eau (Tableau 3). Plusieurs facteurs pourraient influencer la teneur en eau et en matière sèche des plantes comme la nature des fibres, l’âge des plantes, l'état du sol et la durée de conservation du végétal après récolte.

Tableau 3 : Teneur en eau (H \%) et en matière sèche (MS \%) des deux espèces de lavande étudiées :

\begin{tabular}{|c|c|c|}
\hline Espèce & H\% & MS\% \\
\hline Lavandula stoechas spp. stoechas & $\mathbf{6 4 , 0 0 \pm 0 , 3 2}$ & $\mathbf{3 6 , 0 0 \pm 0 , 3 2}$ \\
\hline Lavandula dentata spp.dentata & $\mathbf{6 2 , 6 0 \pm 0 , 7 9}$ & $\mathbf{3 7 , 4 0 \pm 0 , 7 9}$ \\
\hline
\end{tabular}

\section{Rendement des extraits aqueux bruts :}

Le rendement de l'extraction varie en fonction de l'espèce végétale et de son contenu en métabolites, de l'organe utilisé dans l'extraction, les conditions de séchage et de la nature du solvant utilisé dans l'extraction. 
Les résultats des extraits bruts de la partie aérienne des deux lavandes étudiées montrent que les rendements les plus élevés sont ceux obtenus par le mode d'extraction "infusion", notamment chez L. stoechas ; la macération, elle, donne les plus faibles rendements, surtout avec $L$. dentata (Tableau 4). Ainsi, il parait que l’infusion représente le mode de préparation le plus rentable par rapport aux autres modes (Décoction et Macération).

Tableau 4 : Rendement des extraits aqueux bruts des deux espèces étudiées

\begin{tabular}{|c|c|c|c|c|}
\hline \multirow{2}{*}{ Espèce } & Type d'extraction & $\begin{array}{c}\text { Masse } \\
\text { d'extrait } \\
\text { brut en g }\end{array}$ & $\begin{array}{c}\text { Masse sèche de } \\
\text { l'échantillon } \\
\text { végétale en g }\end{array}$ & $\begin{array}{c}\text { Rendement en } \\
\%\end{array}$ \\
\hline \multirow{2}{*}{ L.dentata } & Macération & 0,91 & 10 & $9,1 \pm 0,06$ \\
\cline { 2 - 5 } & Décoction & 1,36 & 10 & $13,6 \pm 0,10$ \\
\cline { 2 - 5 } & Infusion & 1,70 & 10 & $\mathbf{1 7} \pm \mathbf{0 , 1 0}$ \\
\hline \multirow{2}{*}{ L.stoechas } & Macération & 1,34 & 10 & $13,4 \pm 0,12$ \\
\cline { 2 - 5 } & Décoction & 1,53 & 10 & $15,3 \pm 0,06$ \\
\cline { 2 - 5 } & Infusion & 1,84 & 10 & $\mathbf{1 8 , 4} \pm \mathbf{0 , 1 5}$ \\
\hline
\end{tabular}

\section{Rendement en huiles essentielles}

Les extractions par hydrodistillation des parties aériennes de $L$. stoechas et $L$. dentata ont fourni des HEs ayant une coloration variable allant du jaune clair au jaune relativement foncé mais ayant toujours une très forte odeur et persistante.

Des rendements en HEs pouvant être qualifiés de moyens ont été obtenus, soit 2,5\% et $2.9 \%$ respectivement pour L.stoechas et L. dentata (Figure 1)

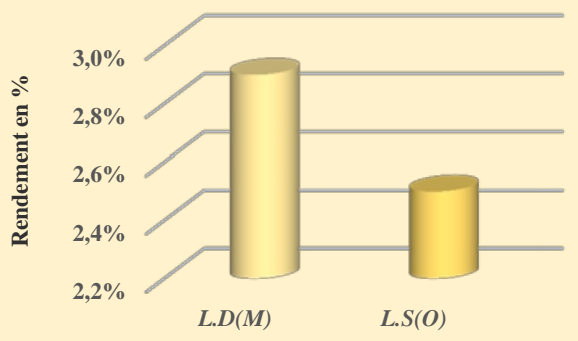

Figure 1 : Rendement en huiles essentielles de L.dentata et L.stoechas avec : L.D(M) : Lavandula dentata de Marrakech et L.S(O) : Lavandula stoechas d'Oulmés

De façon similaire, une étude réalisée par Amarti et al. (2010) sur deux espèces de thym, Thymus ciliatus et Thymus algeriensis a révélé respectivement des rendements en HE de 1,2 et 0,3\%. Les différences des rendements en huiles essentielles entre les espèces, peuvent être dues à diverses conditions, notamment, l'environnement, le génotype et l'origine géographique de la plante (Smallfield, 2001). 


\section{Screening phytochimique}

Dans le but de rechercher les différentes classes des substances secondaires dans les extraits de $L$. stoechas et $L$. dentata, un screening phytochimique a été réalisé via la conduite d'un ensemble de réactions de caractérisation de différents composés chimiques à savoir : les flavonoïdes, les saponines, les tanins, les alcaloïdes, les anthocyanes, les terpènes et les composés réducteurs (Tableau 5). Les résultats de la caractérisation chimique sont classés en fonction des différents critères d'observation que nous avons notés ainsi :

++++ : Réaction très positive; +++ : Réaction positive ; ++ : Réaction moyennement positive ; + : Réaction douteuse ; - : Test négatif.

Tableau 5 : Résultats du screening phytochimique des deux espèces de lavande étudiées

\begin{tabular}{|c|c|c|c|c|}
\hline \multirow{2}{*}{\multicolumn{3}{|c|}{$\begin{array}{c}\text { Métabolites secondaires } \\
\text { Alcaloïdes }\end{array}$}} & L.dentata & L.stoechas \\
\hline & & & - & - \\
\hline \multirow[b]{2}{*}{ Tanins } & \multicolumn{2}{|l|}{ Catéchiques } & +++ & +++ \\
\hline & \multicolumn{2}{|l|}{ Galliques } & ++++ & +++ \\
\hline \multirow{6}{*}{ Flavonoïdes } & \multicolumn{2}{|l|}{ Anthocyanes } & +++ & +++ \\
\hline & \multicolumn{2}{|l|}{ Flavones } & +++ & +++ \\
\hline & \multicolumn{2}{|l|}{ Flavanones } & - & - \\
\hline & \multicolumn{2}{|l|}{ Flavanonols } & - & - \\
\hline & \multicolumn{2}{|c|}{ Leucoanthocyanes } & - & - \\
\hline & \multicolumn{2}{|c|}{ Catéchols } & +++ & +++ \\
\hline \multirow{3}{*}{ Dérivés anthracéniques } & \multicolumn{2}{|c|}{ Anthracéniques libres } & - & - \\
\hline & \multirow{2}{*}{$\begin{array}{l}\text { Anthracéniques } \\
\text { combinés }\end{array}$} & O-hétérosides & - & - \\
\hline & & C-hétérosides & +++ & +++ \\
\hline \multirow{3}{*}{ Composés réducteurs } & \multicolumn{2}{|l|}{ Oses et holosides } & - & - \\
\hline & \multicolumn{2}{|l|}{ Mucilages } & - & - \\
\hline & \multicolumn{2}{|c|}{ Hétérosides cyanogénétiques } & - & - \\
\hline \multirow[b]{2}{*}{ Terpénoïdes } & \multicolumn{2}{|c|}{ Stérols et triterpènes } & ++++ & ++++ \\
\hline & \multicolumn{2}{|c|}{ Saponosides } & - & - \\
\hline
\end{tabular}

Le criblage phytochimique de la partie aérienne des deux lavandes étudiées a mis en évidence la présence de plusieurs composés chimiques réputées avoir des activités biologiques intéressantes (activité antibactérienne, activité antifongique, activité antioxydante...). Il s’agit entre autres des substances polyphénoliques dont les tanins catéchiques et galliques, des flavonoïdes (anthocyanes, flavones et catéchols), des stérols et triterpènes, en plus des anthracéniques combinés (C-hétérosides). Les résultats montrent une similitude entre les deux espèces quant à la présence ou l'absence d'une famille chimique donnée, à l'exception d'une légère différence au niveau des tanins galliques avec une réaction très positive chez $L$. dentata et positive chez $L$. stoechas attestant que la lavande dentée est plus riche en ce métabolite que la lavande stéchade. 


\section{Dosage}

- Teneur en polyphénols totaux et en flavonoïdes des extraits aqueux bruts :

(a)

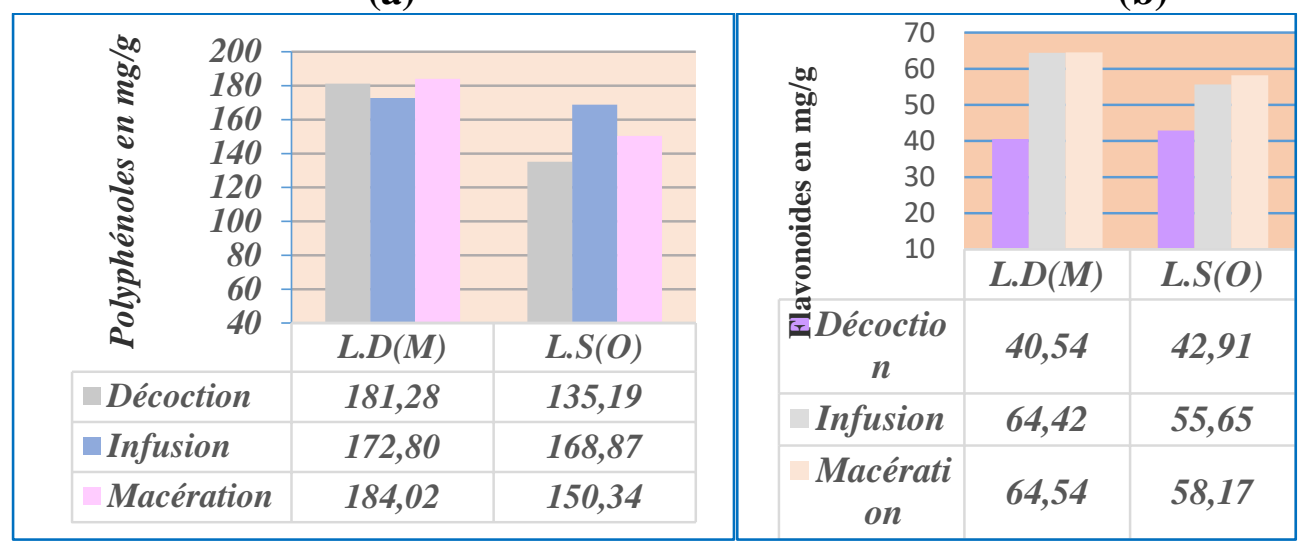

Figure 2 : a : (Teneur en polyphénols des extraits aqueux bruts des lavandes étudiées) et b :( Teneur en flavonoïdes des extraits aqueux bruts des lavandes étudiées).

En présence des polyphénols, le complexe Folin-Ciocalteu voit sa couleur passer du jaune au bleu, ce qui permet de mesurer l'intensité de la couleur à la longueur d'onde de $700 \mathrm{~nm}$ (Huang et al., 2005).

Les résultats représentés dans la Figure 2, a montrent que la teneur en polyphénols diffère selon l'espèce de lavande, le procédé d'extraction, en plus d'une certaine interaction procédé-espèce. Ainsi, il apparait que la lavande dentée est plus riche en polyphénols que la lavande stéchade. De son côté, la macération est le mode le plus efficace pour un meilleur rendement en teneur des polyphénols totaux chez la lavande dentée $(184.02 \mathrm{mg} / \mathrm{g}$ d'extrait), surtout par rapport à l'infusion; en revanche, chez la lavande stéchade, l'infusion représente le mode le plus rentable en polyphénols (168.87 mg/g d'extrait) par rapport à la macération et surtout à la décoction.

Par ailleurs, la densité optique des différents extraits a permis de déterminer les teneurs en flavonoïdes de la partie aérienne de deux plantes étudiées. Les résultats obtenus montrent que chez les deux espèces, l'essentiel de ces métabolites se concentre dans le macéré, le décocté étant lui, le moins riche. Aussi, la lavande dentée est plus riche en flavonoïdes que la lavande stéchade, la teneur la plus importante étant de $64.54 \mathrm{mg} / \mathrm{g}$ dans le macéré (Figure 3, b). 
- Teneur en tanins totaux et en tanins condensés des extraits aqueux bruts :

(c)

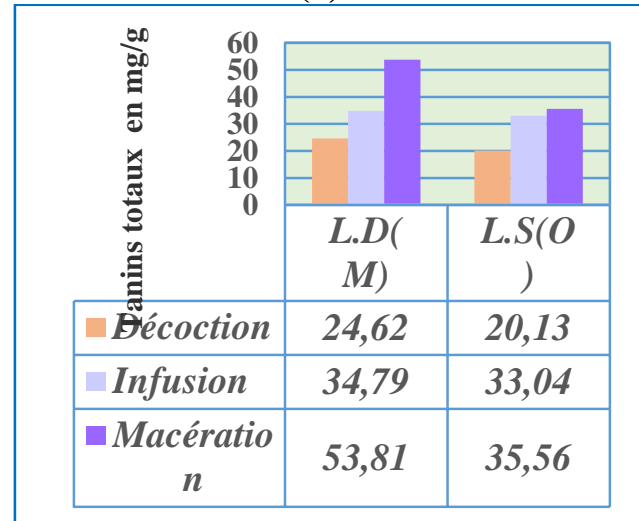

(d)

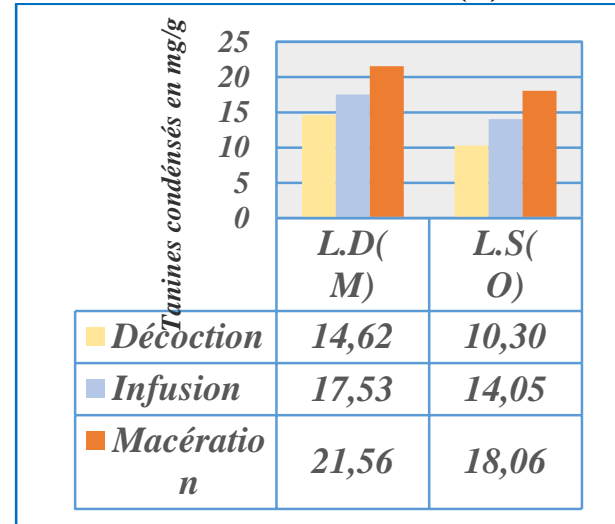

Figure 3 : c : (Teneur en tanins totaux des extraits aqueux bruts des lavandes étudiées) et $\mathbf{d}$ :(Teneur en tanins condensés des extraits aqueux bruts des lavandes étudiées).

Les résultats obtenus pour le dosage des tanins totaux des deux espèces de lavande étudiées sont présentés dans la Figure 3, c. On remarque que $L$. dentata possède des teneurs importantes en tanins totaux, comparée à L.stoechas ; ceux-ci se concentrent principalement dans le macéré avec une valeur maximale notée chez $L$. dentata $(53.8 \mathrm{mg} / \mathrm{g})$. Les mêmes observations peuvent être faites quant au dosage des tanins condensés, reporté dans la Figure 3,d ; l'extraction par macération révèle des teneurs importantes chez L. dentata en comparaison avec l'infusion et la décoction, la teneur la plus élevée étant enregistrée chez $L$. dentata (21.56 mg/g).

D'après ces résultats, on constate clairement que du point de vue quantitatif, il y a une différence entre les deux espèces quant à leur teneur en polyphénols (flavonoïdes, tanins totaux et tanins condensés) qui pourrait être liée aux facteurs environnementaux comme la sécheresse, le sol et les maladies (Ebrahimi et coll, 2008). De même, la période de récolte, le stade de développement de la plante, la méthode d'extraction et de quantification peuvent également influencer la teneur en phénols totaux (Lee et coll, 2003).

D’une façon similaire en 1992, une importante étude menée par GarcíaVallejo a montré une différence entre certaines espèces de lavande sauvages issues de différentes régions d'Espagne, Cependant aucune étude n'a été faite pour les espèces du genre Lavandula, pour faire une corrélation entre la composition chimique et spécificités génétiques (Benabdelkader, 2014). Par ailleurs, il faut signaler que des variations intra-spécifiques chez $L$. dentata récoltée dans différents endroits en Algérie (Bousmaha et al., 2006), tant qualitatives (composés différents) que quantitatives (proportions différentes de certains composés) ont été mentionnées, elles peuvent être dues à des 
différences climatiques, géographiques ou saisonnières, mais, elles peuvent avoir aussi une origine génétique (Benabdelkader, 2014).

En outre, il apparait que le mode de préparation des extraits a une grande influence sur la concentration des composés chimiques ; en effet, la macération, comparée à la décoction et à l'infusion représente généralement le mode le plus rentable en polyphénols, flavonoïdes, tanins totaux et tanins condensés. Or, d'après des investigations ethnobotaniques menées par Bachiri et al., 2015 auprès d'herboristes et vendeurs de plantes sur les deux lavandes, sujettes du présent travail, c’est le décocté qui est utilisé pour préparer la majorité des remèdes, ceci, tant à Marrakech . Cela soulève d'une part le degré d'efficacité de la phyto médication par ces lavandes, l'extrait utilisé n’étant pas le meilleur et d'autre part la nécessité de mener des études scientifiques pour valider ou rejeter certaines pratiques ancrées dans les traditions médicinales.

Pouvoir antibactérien des huiles essentielles et des extraits bruts

- Activité antibactérienne des huiles essentielles

La méthode de diffusion des disques en milieu gélosé a permis de mettre en évidence le pouvoir antibactérien des huiles essentielles de $L$. dentata et $L$. stoechas vis-à-vis de quatre bactéries ( $E$. coli, K. pneumoniae, $S$. aureus et $P$. mirabilis). Les volumes d'huiles essentielles utilisées furent : 5, 10, 15 et $20 \mu \mathrm{l}$. Dans le Tableau 4, sont reportés les diamètres d'inhibition et les antibiotiques de référence utilisés.

Tableau 4 : Diamètres des zones d'inhibition $(\mathrm{mm})$ illustrant l'activité antibactérienne des huiles essentielles issues des deux lavandes étudiées

\begin{tabular}{|c|c|c|c|c|c|}
\hline \multirow[t]{2}{*}{ Espèce } & \multirow{2}{*}{$\begin{array}{c}\text { Concentrations } \\
(\mu \mathrm{l})\end{array}$} & \multicolumn{4}{|c|}{ Zones d'inhibition (mm) } \\
\hline & & S.aureus & E.coli & K.pneumoniae & P.mirabilis \\
\hline \multirow{4}{*}{ L.D } & 5 & $18 \pm 0,29$ & $8 \pm 0,10$ & $8 \pm 0,15$ & $9 \pm 0,10$ \\
\hline & 10 & $20 \pm 0,32$ & $9 \pm 0,20$ & $9 \pm 0,00$ & $10 \pm 0,06$ \\
\hline & 15 & $25 \pm 0,00$ & $10 \pm 0,21$ & $11 \pm 0,21$ & $13 \pm 0,32$ \\
\hline & 20 & $30 \pm 0,00$ & $12 \pm 0,29$ & $12 \pm 0,00$ & $14 \pm 0,15$ \\
\hline \multirow{4}{*}{ L.S } & 5 & NA & $8 \pm 0,12$ & $7 \pm 0,50$ & $8 \pm 0,26$ \\
\hline & 10 & NA & $9 \pm 0,00$ & $10 \pm 0,26$ & $9 \pm 0,40$ \\
\hline & 15 & NA & $11 \pm 0,29$ & $12 \pm 0,21$ & $10 \pm 0,55$ \\
\hline & 20 & NA & $12 \pm 0,15$ & $12 \pm 0,25$ & $10 \pm 0,25$ \\
\hline \multicolumn{2}{|r|}{ ATB } & $\begin{aligned} & \mathrm{CN}(\mathrm{S}) \\
= & 19 \pm 0,00\end{aligned}$ & $\begin{array}{c}\text { IMP (S) } \\
=26 \pm 1,00\end{array}$ & $\begin{array}{c}\text { IMP (S) } \\
=26 \pm 0,00\end{array}$ & $\begin{array}{c}\text { IMP (S) } \\
=26 \pm 1,00\end{array}$ \\
\hline
\end{tabular}

NA : Non Actif ; ATB : antibiotique de référence ; $\mathrm{CN}_{30}$ : Gentamicine (30 $\left.\mu \mathrm{g} / \mathrm{disc}\right)$; $\mathrm{IPM}_{10}$ : Imipenème (10 $\left.\mathrm{\mu g} / \mathrm{disc}\right)$

On peut ainsi constater qu'à l'exception de l'HE de L. stoechas qui ne montre aucun effet sur $S$. aureus Tableau 4, les huiles essentielles extraites à partir de la partie aérienne des deux espèces étudiées exercent une activité inhibitrice modérée de la croissance vis-à-vis des bactéries Gram (-) (E. coli, K. pneumoniae et $P$. mirabilis) car le diamètre de zone d'inhibition 
est inférieure à $15 \mathrm{~mm}$ (référence des standards des aromatogrammes). Les Gram (+), dans le cas du $S$. aureus, montrent une sensibilité remarquable à $L$. stoechas (Figure 4) et cette activité augmente avec le volume de l'huile essentielle déposée sur le disque.

(A)

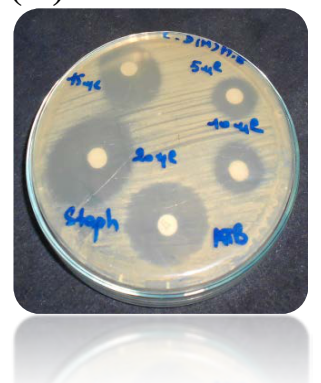

(B)

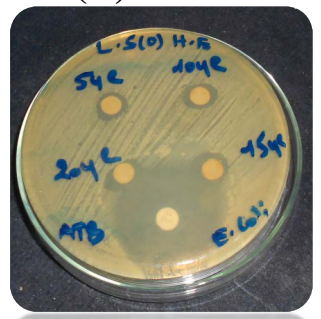

(C)

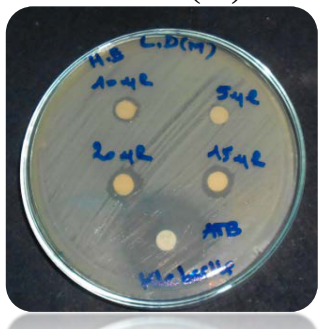

(D)

Figure 4 : Effet inhibiteur des huiles essentielles des deux espèces de lavande étudiées sur les souches bactériennes testées

A : Effet de l'HE de L.D sur S. aureus ; B : Effet de l'HE de L.S sur E. coli ; C : Effet de l'HE de L.D sur K. pneumoniae ; D : Effet de l'HE de L.D sur E coli

- Activité antibactérienne des extraits aqueux bruts

D'un autre part, l'évaluation de l'activité antibactérienne des extraits aqueux bruts a été réalisée également par la technique d'aromatogramme. Les volumes des extraits déposés sur les disques furent de 5, 10, 15, 20 et 25 $\mu l$. Le témoin négatif utilisé fut l'eau distillée stérile. La figure 5 regroupe les résultats de l'activité antibactérienne des extraits aqueux bruts des deux plantes.

S. aureus n'a montré aucune sensibilité vis-à-vis de tous les extraits testés et aucune zone d'inhibition n’a été détectée. Seul le macéré de $L$. stoechas a pu avoir un effet remarquable sur K. pneumoniae représenté par une zone d'inhibition ( $15 \mathrm{~mm}$ ) de diamètre (figure 5-a).

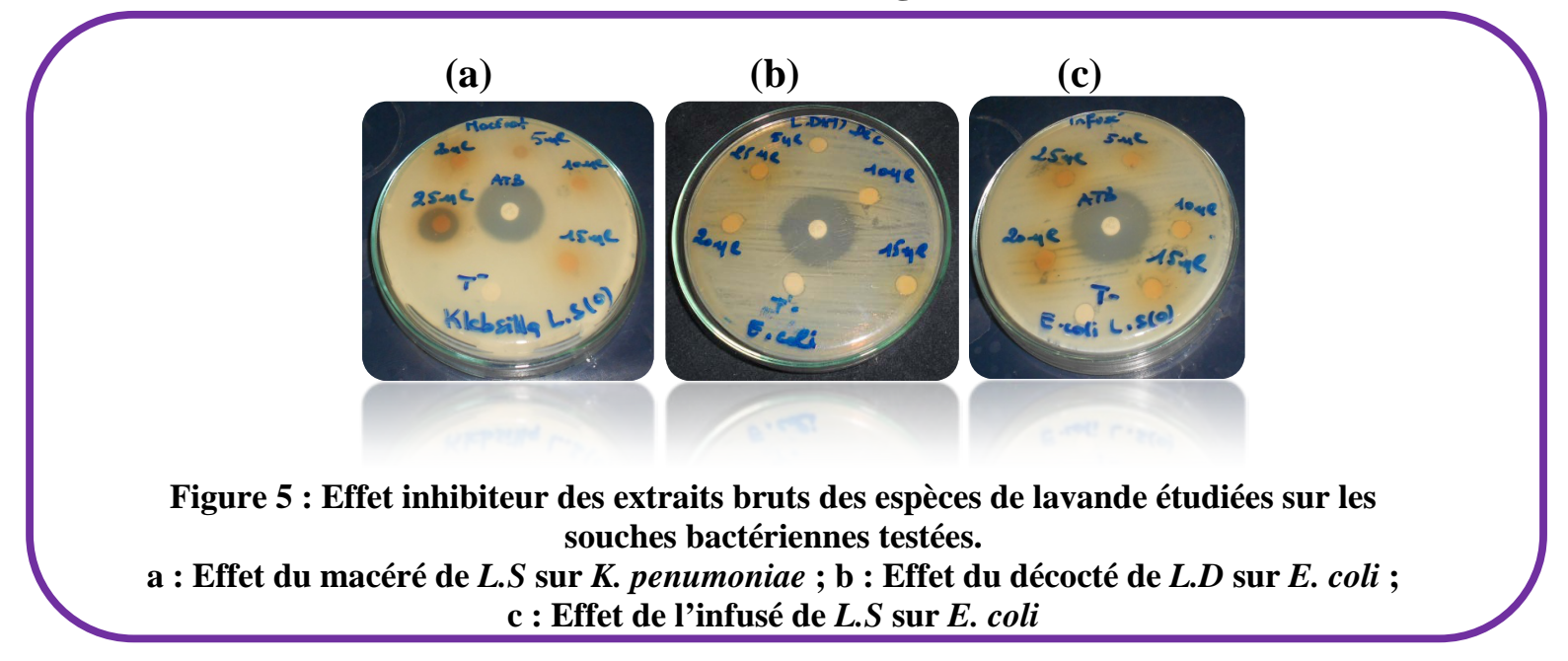


Par ailleurs la partie aérienne de $L$. dentata dont la richesse en huiles essentielles est démontrée précédemment a une activité très efficace sur les bactéries Gram (+) et plus ou moins modérée sur les Gram (-) ; cette activité inhibitrice dépasse largement l'effet de l'antibiotique de référence à partir du volume $15 \mu \mathrm{l}$ de l'huile essentielle pour $S$. aureus. Ces résultats corroborent ceux mentionnés par d'autres chercheurs, notamment Miladinovic et al. (2012) rapportant que les huiles essentielles de certaines lavandes manifestent une activité supérieure aux antibiotiques conventionnels envers S. aureus.

Cependant, l'activité antibactérienne de l'huile essentielle de la lavande stoechade s'est avérée moins active par rapport à la lavande dentée contre les bactéries testées. Cette activité contre les Gram (-) (E. coli, K. pneumoniae et $P$. mirabilis) n'est détectée qu'à partir des disques de charge (10 $\mu \mathrm{l}$ de l'huile essentielle), par contre aucune zone d'inhibition n'a été observée contre la bactérie Gram (+) S.aureus.

Les mécanismes probables du pouvoir antimicrobien des huiles essentielles de la lavande dentée contre les bactéries à Gram (+) sont basés sur le contact direct de leurs composés hydrophobes avec la membrane cellulaire, et leur pouvoir de répartir les phospholipides en les rendant plus perméables ce qui pourrait causer des dommages structuraux ou une rupture complète des membranes cellulaires et des pertes de nutriments (Pieroza $\boldsymbol{e t}$ al., 2009).

La différence d'activité des huiles issues de différentes espèces est due principalement aux conditions climatiques et édaphiques des deux localités, par conséquent ces facteurs ont une influence sur la composition chimique d'huile essentielle de la plante et leur activité biologique. On peut conclure que c'est l'essence de Lavandula dentata qui a manifesté le plus grand pouvoir antibactérien par rapport à celle de Lavandula stoechas ; ces résultats corroborent ceux trouvés par d'autres chercheurs, notamment Ghanmi et al., (2011) .Un travail semblable réalisé sur deux espèces du genre Thymus montre aussi que l'huile essentielle de Thymus ciliatus (Desf.) Benth a une forte activité antibactérienne en comparaison à Thymus algeriensis (Amarti et al., 2010)

Concernant l'activité antibactérienne des extraits aqueux bruts, on constate qu'il y a une corrélation inverse entre le test de dosage et l'activité antibactérienne, les extraits les plus riches en flavonoïdes ont une activité modérée par rapport aux autres Figure 2, b et Figure 5.

Ces résultats ne sont pas en concordent avec ceux d'autres chercheurs, notamment Iinuma et al., (1994) ; Haraguchi et al., (1998) ; Iniesta-Sanmartin et al., (1990) et Tim et al., (2005) qui ont rapporté que les espèces riches en flavonoïdes possèdent une activité antimicrobienne ; 
ces métabolites sont considérés comme de très bons agents antimicrobiens (Harborne et al., 2000).

\section{Détermination des CMI et CMB}

- Cas des huiles essentielles et des extraits :

Les valeurs des CMI et CMB ainsi que le pouvoir antibactérien des huiles essentielles extraites des deux espèces de lavande étudiées sont présentées dans le Tableau 5.

Tableau 5 : Concentrations minimales inhibitrices (CMI) et bactéricides (CMB) des huiles essentielle des quatre plantes étudiées et leur pouvoir antibactérien

\begin{tabular}{|c|c|c|c|c|c|}
\hline $\begin{array}{l}\text { Nom de } \\
\text { l'espèce }\end{array}$ & $\begin{array}{c}\text { Souches } \\
\text { bactériennes }\end{array}$ & $\begin{array}{c}\text { CMB } \\
(\mathrm{mg} / \mathrm{ml})\end{array}$ & $\begin{array}{c}\text { CMI } \\
(\mathrm{mg} / \mathrm{ml})\end{array}$ & CMB/CMI & Pouvoir antibactérien \\
\hline \multirow{4}{*}{ L.D } & S.aureus & 0,10 & 0,08 & 1,25 & Bactéricide \\
\hline & E.coli & 0,16 & 0,14 & 1,14 & Bactéricide \\
\hline & K.pneumoniae & 0,16 & 0,14 & 1,14 & Bactéricide \\
\hline & P.mirabilis & 0,16 & 0,14 & 1,14 & Bactéricide \\
\hline \multirow{4}{*}{ L.S } & S.aureus & 0,23 & 0,17 & 1,35 & Bactéricide \\
\hline & E.coli & 0,16 & 0,14 & 1,14 & Bactéricide \\
\hline & K.pneumoniae & 0,16 & 0,14 & 1,14 & Bactéricide \\
\hline & P.mirabilis & 0,16 & 0,14 & 1,14 & Bactéricide \\
\hline
\end{tabular}

D’après ces résultats, le rapport CMB/CMI est compris entre 1.14 et 1.4 pour les huiles essentielles et entre 1 et 1,36 pour les extraits aqueux bruts Tableau 6. En se référant à Canillac et Mourey 2001 qui ont mentionné que le rapport $\mathrm{CMB} / \mathrm{CMI}$ doit être inférieur à 4 pour confirmer que le pouvoir bactérien d'une huile essentielle ou d'un extrait est bactéricide, nous pouvons conclure que les huiles essentielles et les extraits des deux espèces ont un pouvoir bactéricide vis-à-vis des souches utilisées (Figures 6).

Tableau 6 : Concentrations minimales inhibitrices (CMI) et bactéricides (CMB) et pouvoir antibactérien des extraits bruts de $L$. dentata et $L$. stoechas

\begin{tabular}{|c|c|c|c|c|c|c|}
\hline \multirow[t]{6}{*}{ Espèce } & $\begin{array}{c}\text { Mode de } \\
\text { préparation }\end{array}$ & $\begin{array}{c}\text { Souches } \\
\text { bactériennes }\end{array}$ & $\begin{array}{c}\text { CMB } \\
(\mathrm{mg} / \mathrm{ml})\end{array}$ & $\begin{array}{c}\text { CMI } \\
(\mathrm{mg} / \mathrm{ml})\end{array}$ & CMB/CMI & $\begin{array}{c}\text { Pouvoir } \\
\text { antibactérien }\end{array}$ \\
\hline & & S.aureus & 32 & 24,5 & 1,30 & Bactéricide \\
\hline & & E.coli & 50 & 40,5 & 1,23 & Bactéricide \\
\hline & Infusion & K.pneumoniae & 50 & 40,5 & 1,23 & Bactéricide \\
\hline & & P.mirabilis & 32 & 32 & 1 & Bactéricide \\
\hline & & S.aureus & 32 & 24,5 & 1,30 & Bactéricide \\
\hline \multirow[t]{10}{*}{ L.D } & & E.coli & 40,5 & 40,5 & 1 & Bactéricide \\
\hline & Décoction & K.pneumoniae & 32 & 32 & 1 & Bactéricide \\
\hline & & P.mirabilis & 32 & 32 & 1 & Bactéricide \\
\hline & & S.aureus & 50 & 40,5 & 1,23 & Bactéricide \\
\hline & & E.coli & 40,5 & 32 & 1,26 & Bactéricide \\
\hline & Macération & K.pneumoniae & 40,5 & 32 & 1,26 & Bactéricide \\
\hline & & P.mirabilis & 40,5 & 32 & 1,26 & Bactéricide \\
\hline & & S.aureus & 40,5 & 32 & 1,26 & Bactéricide \\
\hline & & E.coli & 60,5 & 50 & 1,21 & Bactéricide \\
\hline & Infusion & K.pneumoniae & 60,5 & 50 & 1,21 & Bactéricide \\
\hline
\end{tabular}




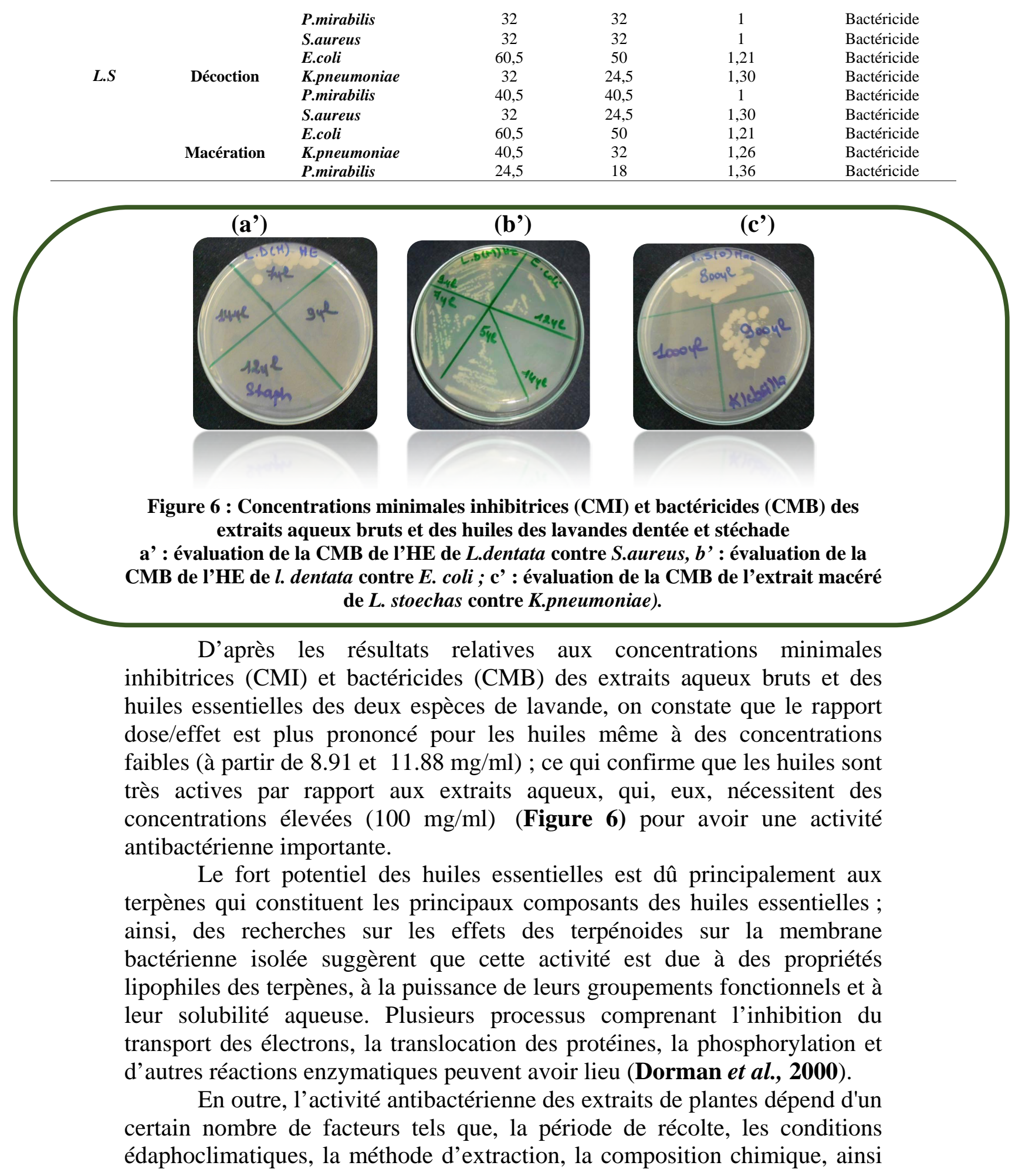


que les types de microorganismes testés et les conditions de réalisation des tests (Al-Reza et al., 2010 ; Obeidat et al., 2012).

\section{CONCLUSION}

Ce travail entre dans le cadre de la valorisation des plantes aromatiques et médicinales marocaines; nous nous sommes intéressés à l'étude comparative interspécifique de lavande ( $\boldsymbol{L}$. dentata de Marrakech, L. stoechas d'Oulmès) au niveau de la composition chimique, du point de vue qualitatif et quantitatif, le rendement en huiles essentielles et en extraits aqueux bruts, et aussi l'activité antibactérienne vis-à-vis de certaines souches bactériennes pathogènes.

Les résultats du screening phytochimique de la partie aérienne des deux lavandes étudiées ont révélé une similitude quant à leur composition qualitative avec une légère différence au niveau des tanins galliques.

Par ailleurs, le dosage des extraits a révélé que la lavande dentée renferme des teneurs en polyphénols, en flavonoïdes, en tanins totaux et condensés plus élevées que la lavande stoechade.

La détermination des rendements des extraits aqueux bruts a permis de retenir le mode d'extraction le plus efficace pour chaque espèce ; il s'agit de l'infusion, suivie de la décoction et de la macération, avec un rendement important noté chez l'infusé de L. stoechas. Aussi, le rendement d'extraction des huiles essentielles par hydrodistillation a montré une rentabilité élevée chez L. dentata.

Par ailleurs, l'activité antibactérienne de l'huile essentielle de la lavande dentée a montré un effet antibactérien important contre les quatre souches testées avec une efficacité impressionnante vis-à-vis de la bactérie Gram (+) S.aureus. De leur côté, l’infusé et le macéré de L. stoechas ont montré un effet modéré contre $E$. coli et $K$. pneumoniae respectivement, et pour le décocté de $L$. dentata contre $E$. coli. Les bactéries Gram (+) se sont montrées non sensibles vis-à-vis de tous les extraits testés et aucune zone d'inhibition n'a été détectée.

En fin, le pouvoir bactéricide des huiles des deux espèces à des concentrations faibles a été confirmé, ce qui est en faveur d'une efficacité plus élevée, quant à celui des extraits aqueux bruts, il s'est manifesté à des concentrations élevées. Les huiles essentielles des deux lavandes étudiées sont donc plus efficaces que leurs extraits aqueux.

\section{References:}

1. Al-Reza, S.M., Rahman, A., and Kang S.C. Inhibition of plant pathogens in vitro and in Vivo with essential oil and organic extracts of Cestrum nocturnum L: Pesticide biochemistry and Physiology, Vol 96: 86-92, 2010. 
2. Amarti ,F., Satrani, B., Ghanmi, M.Farah ,A., Aafi,A., Aarab,L., El Ajjouri, M., et Chaouch,A . Composition chimique et activité antimicrobienne des huiles essentielles de Thymus algeriensis Boiss. \& Reut et Thymus ciliatus (Desf.) Benth du Maroc, Biotechnol. Agron. Soc. Environ.14(1), 141-148, 2010.

3. Ba,K. Tine,E., Destain, J., Cisse, N., et Thonart P. Étude comparative des composés phénoliques, du pouvoir antioxydant de différentes variétés de sorgho sénégalais et des enzymes amylolytiques de leur malt. Biotechnol. Agro. Soc. Environ. Vol. 14. pp. 131-139,2010.

4. Bachiri, L.,Labazi ,N., Daoudi ,A., Ibijbijen, J.,Nassiri, L., Mokhtari, F., et Echchegadda G. Etude ethnobotanique de quelques lavandes marocaines spontanées Int. J. Biol. Chem. Sci. 9(3) : 1308-1318, 2015.

5. Bekro,Y.A., Mamyrbekova,J.A., Boua,B.B., Ehile, E.E. Étude Ethnobotanique Et Screening Phytochimique de Caesalpinia Benthamiana (Baill.) Herend. rt Zarucchi (Caesalpiniaceae). Sciences \& Nature: Vol. 4, No. 2, 217-225, 2007.

6. Bellakhdar, J. La pharmacopée marocaine traditionnelle, ibis Press, Paris, Edition Ibis Press p : 759, 1997.

7. Benabdelkader,T. Biodiversité, Bioactivité et Biosynthèse des Composes Terpéniques Volatils des Lavandes Ailées, Lavandula stoechas Sensu Lato, un Complexe d’Espèces Méditerranéennes d'Intérêt Pharmacologique, thèse en biologie et Ecophysiologie végétale de l'Ecole Normale Supérieure de Kouba-Alger, Algérie \& de l’Université Jean-Monet de Saint-Etienne, France. 259 p, 2014.

8. Bousmaha,L., Boti, J.B. Infraspecific chemical variability of the essential oil of Lavandula dentata L. from Algeria. Flavour Fragr. J. 21, 368-372, 2006.

9. Bruneton,J .Pharmacognosie, Phytochimie, Plantes médicinales,2éme édition, Technique et Documentation, Lavoisier, Paris, p. 915, 1993.

10. Canillac,N., and Mourey,A. Antibacterial activity of the essential oil of Picea excelsa on Listeria, Staphylococcus aureus and coliform bacteria. Food Microbiol. 18 : pp. 261-268, 2001.

11. Chu, C.J., Kemper,K.J. Lavender (Lavandula spp.). Longwood Herbal Task Force; 32 p, 2001

12. Clevenger, J.F. Apparatus for the Determination of Volatile Oil. Journal of the American Pharmaceutical Association: Vol. 17, No. 4,345-349, 1928. 
13. Dohou,N. Screening phytochimique d’une endémique ibéromarocaine thymelaea lythroides, Bull. Soc. Pharm. Bordeaux, 2003,142, 61-78, 2003.

14. Dorman,H.J.D., and Deans,S.G. Antimicrobial agents from plants: antibacterial activity of plant volatile oils. 88 , p: 308-316, 2000.

15. Dupont,F. ,Guignard,J.L. Abrégés de pharmacie. Botanique, les familles de plantes. (15e édn). Elsevier Masson: Paris, 2012.

16. Ebrahimi,N.S., Hadian, J., Mirjalili ,M.H., Sonboli, A., et Yousefzadi, M. Essential oil composition and antimibacterial activity of Thymus caramanicus at different phonological stages. Food chemistry, 110: 927-931, 2008.

17. Fennane, M., Ibn Tattou,M., Ouyahya,A., \& El Oualidi J.Flore Pratique du Maroc, vol. 2. Angiospermae ( Leguminosae Lentisbulariaceae) Trav. Inst. Sci., sér. Bot. 38.Rabat, 636p, 2007

18. Garcia-Vallejo,M.I., Aceites esenciales de las Lavandulas ibéricas: ensayo de la quimiotaxonomía. Departamento de biologia vegetal I. Madrid, Universidad Complutense de Madrid. PhD : 498p, 1992.

19. Ghanmi,M ., Satrani,B., Aberchane,M., Rchid,M.I., Aafi,A., et El abid.,A. Plantes Aromatiques et Médicinales au Maroc : Les milles et une vertu, Centre de recherche forestier Maroc, Haut-Commissariat aux Eaux et Forêts et lutte contre la désertification, Edition collection Maroc Nature, P 310, 2011.

20. Gören, A.C., Topçu, G., Bilsela,G., Bilsela, M., Aydoğmus, Z., et Pezzuto J.M.Z. Naturforsch. 57c 797-800, 2002.

21. Haraguchi,H., Tanimoto, K., Tamura, Y., Mizutani,K., and Kinoshito, T. Mode of antibacterial action of retrochalcones from Glycyrrhizainflata. Phytochemistry. 48 : 125-129, 1998.

22. Harborne, J.B., and Williams, C.A. Advances in flavonoid research since 1992. Phytochemistry, 55, 481-504, 2000.

23. http://www.iaea.org/programmes/nafa/d3/index.html

24. Huang .Perfumer and flavorist, Vol. 13, $\mathrm{N}^{\circ} 2$, 67p, 1987. In Besombes C. Contribution à l'étude des phénomènes d'extraction hydro-thermo-mécanique d'herbes aromatiques. Applications généralisées. Thèse de doctorat. Université de La Rochelle, 41p, 2008.

25. Iinuma, M., Tsuchiya, H., Sato, M., Yokoyama, J., Ohyama M., Ohkawa, Y., Tanaka, T., Fujiwara, S. and Fujii, T. Flavanones with antibacterial activity against Staphylococcus aureus. J. Pharm. Pharmacol. 46: 892-895, 1994.

26. Iniesta-Sanmartin E., Barberan F.A.T., Guirado A. and Lorents F.T. Antibacterial flavonoids from Helichrysumpicardii and $\mathrm{H}$. italicum. Planta Medica. 56: 648 649, 1990. 
27. Kuete, V., Penlap Beng,V., Etoa,f-X., Modjo, S.L., Bogne, P., Assob, J.C., et Lontsi ,D. Activité antimicrobienne de l'extrait total et des fractions de jus de fruits de Citrus medical in (Rutaceaa). Pharm. Méd. Trad. Afr. Vol. 13, 91-101, 2004.

28. Kumaran,A., et karunakaran,R.J. Antioxidant and free radical scavenging activity of an aqueous extract of Coleus aromaticus. Food chemistry.97: 109-114, 2005.

29. Lee, K.W., Kim Y.J., Lee H.j., et Lee, C.Y. Cocao has more phenolic phytochemicals and a higher antioxidant capacity than teas and red wine. Food chemistry, 51: 7292-7295, 2003.

30. Miladinovic, D., Ilic, B., Mihajilov-Krestv, T.,Nikolic, N., Miladinovicl,. \& Cvetkovic,O. Investigation of the chemical composition antibacterial activity relationship of essential oils by chemometric methods.Analytical and bioanalytical chemistry.403:1007-18,2012.

31. Obeidat,M., Shatnawi,M. , Al-alawi,M., Al-Zu’bi E., Al-Dmoor H., Al-Qudah M.,and El-Qudah I. Antimicrobial activity of crude extracts of some plant leaves: Research Journal of Microbiology, Vol 7: 59-67, 2012.

32. Pieroza, K.M. Chemical characterization and antimicrobial activity of essential oils of salvia L. species; Ciênc. Tecnol. Aliment, Campinas, 29(4) : 764-770, out.-dez, 2009.

33. Ponce A.G., Fritz R., del Valle C., et Roura S.I. Antimicrobial activity of essential oils on the native microflora of organic Swiss chard. Lebensm.-Wiss.u.-Technol.36, pp.679-684, 2003.

34. Rhayour, K. Etude du mécanisme de l'action bactéricide des huiles essentielles sur Esherichia coli, Bacillus subtilis et sur Mycobacterium phlei et Mycobacterium fortuitum. Thèse de doctorat. Université Sidi Mohamed Ben Abdellah. Fès, Maroc, 170 p, 2002. Schroeder M.P and Messing A.M., Methods for comparing the antibacterial activity of essential oils and other aqueous insoluble compounds, Bull Nat. Formulary Comm, 17, 213-218, 1949.

35. Smallfield.B Introduction to growing herbs for essential oils, medicinal and culinary purposes. Crop et Food Research (45): 1-4, 2001.

36. Sqalli,H., El Ouarti,A., Ennabili,A., Ibnsouda,S., Farah,A., Haggoud, A., et Iraqui, M. Evaluation de l'effet antimycobactérien de plantes du centre-nord du Maroc. Bull Soc Pharm, 146, 271,-288, 2007.

37. Tim, C.T.P., and Andrew, J. L. Antimicrobial activity of flavonoids. Int. J. Antimicrob. Ag. 26:343-356, 2005. 
38. Twidwell, E.K., Wagner, J.J., and Thies Nancy, J. Use a Microwave Oven to Determine Moisture Content of Forages. 8077p, 2002.

39. Upson, T., Andrews, S. The Genus Lavandula. Portland and Oregon. Timber Press: USA, 2004.

40. Vermerris,W., Nicholson, R. Isolation and Identification of Phenolic Compounds, Phenolic Compound Biochemistry. Dordrecht: Springer. p: 1-70,2006. 